\section{References}

1. Reddy MA, Francis PJ, Berry V, Bhattacharya SS, Moore AT. Molecular genetic basis of inherited cataract and associated phenotypes. Surv Ophthalmol. 2004;49:300-15.
2. Shiels A, Bennett T, Hejtmancik M, Fielding J. Cat-Map: putting cataract on the map. Mol Vision. 2010;16:2007-15.

\title{
Is post-operative perfluorocarbon liquid tamponade for macula-on giant retinal tear safer than silicone oil?
}

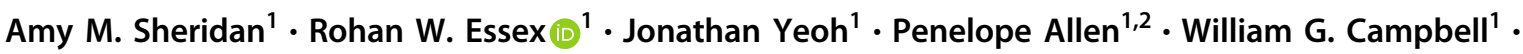 \\ Thomas L. Edwards (1) ${ }^{1,2}$
}

Received: 5 August 2018 / Revised: 25 September 2018 / Accepted: 26 September 2018 / Published online: 7 December 2018

(c) The Royal College of Ophthalmologists 2018

The incidence of unexplained central vision loss immediately following removal of silicone oil (ROSO) has been reported at between $3.3 \%$ [1] and 5.9\% [2], but may be considerably higher in certain retinal detachment subgroups. For example, the rate of ROSO maculopathy after macula-on giant retinal tear (GRT) repair was reported as high as $50 \%$ [1] in this journal, perhaps suggesting maculaon GRTs (Fig 1), or indeed any macula-on retinal detachment, are uniquely susceptible to ROSO maculopathy.

Perfluorocarbon heavy liquid (PFCL) as a short-term post-operative tamponade agent in GRT repair is a safe and effective alternative to silicone oil ( $\mathrm{SiO})$ or gas. This technique, first described by Bottoni [3] and subsequently by others [4-7], is used to manage all GRT detachments at the Royal Victorian Eye and Ear Hospital. PFCL remains in the eye for approximately 14 days, before exchange with fluid, air or gas. To investigate whether removal of short-term PFCL tamponade resulted in a lower rate of unexplained vision loss than ROSO in macula-on GRT detachments, we performed a consecutive retrospective review of all maculaon GRT repairs between 19 August 2007 and 12 December 2016. Best-corrected visual acuity (VA) was recorded at initial presentation, and 3 months following PFCL removal. The outcome of the procedure was determined at 3 months.

Thomas L. Edwards

thomas.edwards@unimelb.edu.au

Royal Victorian Eye and Ear Hospital, Melbourne, Australia

2 Centre for Eye Research Australia, Royal Victorian Eye and Ear Hospital, Melbourne, Australia
Statistical analysis was performed using a paired student's $t$ test assuming equal variance (alpha $=0.05$ ).

A total of 25 eyes in 24 patients (mean age 57 years; range 39-79 years) comprising 4 female patients (16.7\%) and 20 male patients $(83.3 \%)$ were included in the study cohort (Table 1). The mean (range) duration of PFCL tamponade was 14.6 days, (10-28 days) before removal and exchange either with 20 or $25 \%$ SF6 gas $(n=13,52 \%)$, air $(n=7$, $28 \%)$ or balanced salt solution $(n=5,20 \%)$. The mean baseline VA was 76 letters. The mean VA 3 months post-

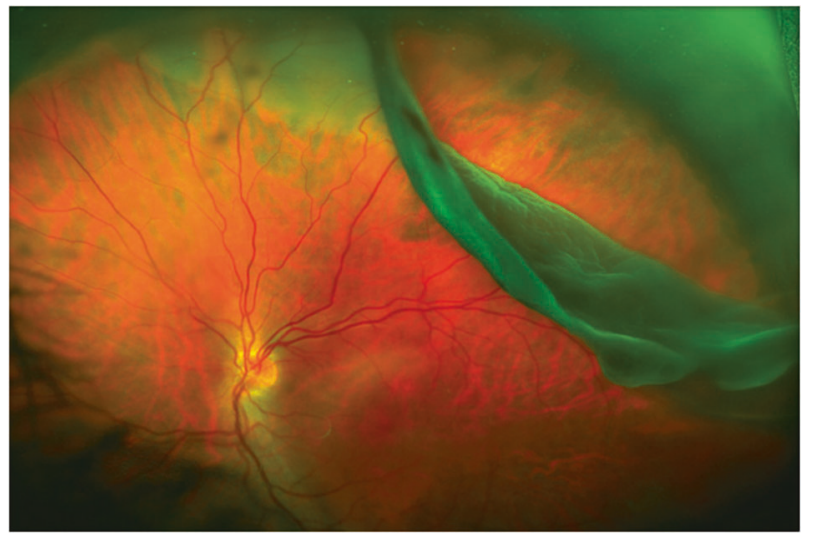

Fig. 1 Wide-field fundus photograph of the left eye showing a superotemporal giant retinal tear (GRT). A characteristic feature of this type of retinal detachment is posterior folding of the detached retina. Repositioning of the fold is facilitated by intra-operative perfluorocarbon liquid (PFCL) tamponade. Intra- or post-operative slippage can occur when the PFCL is exchanged for gas or, more rarely, silicone oil. An effective alternative is short-term post-operative tamponade with PFCL, which minimises the risk of retinal slippage 


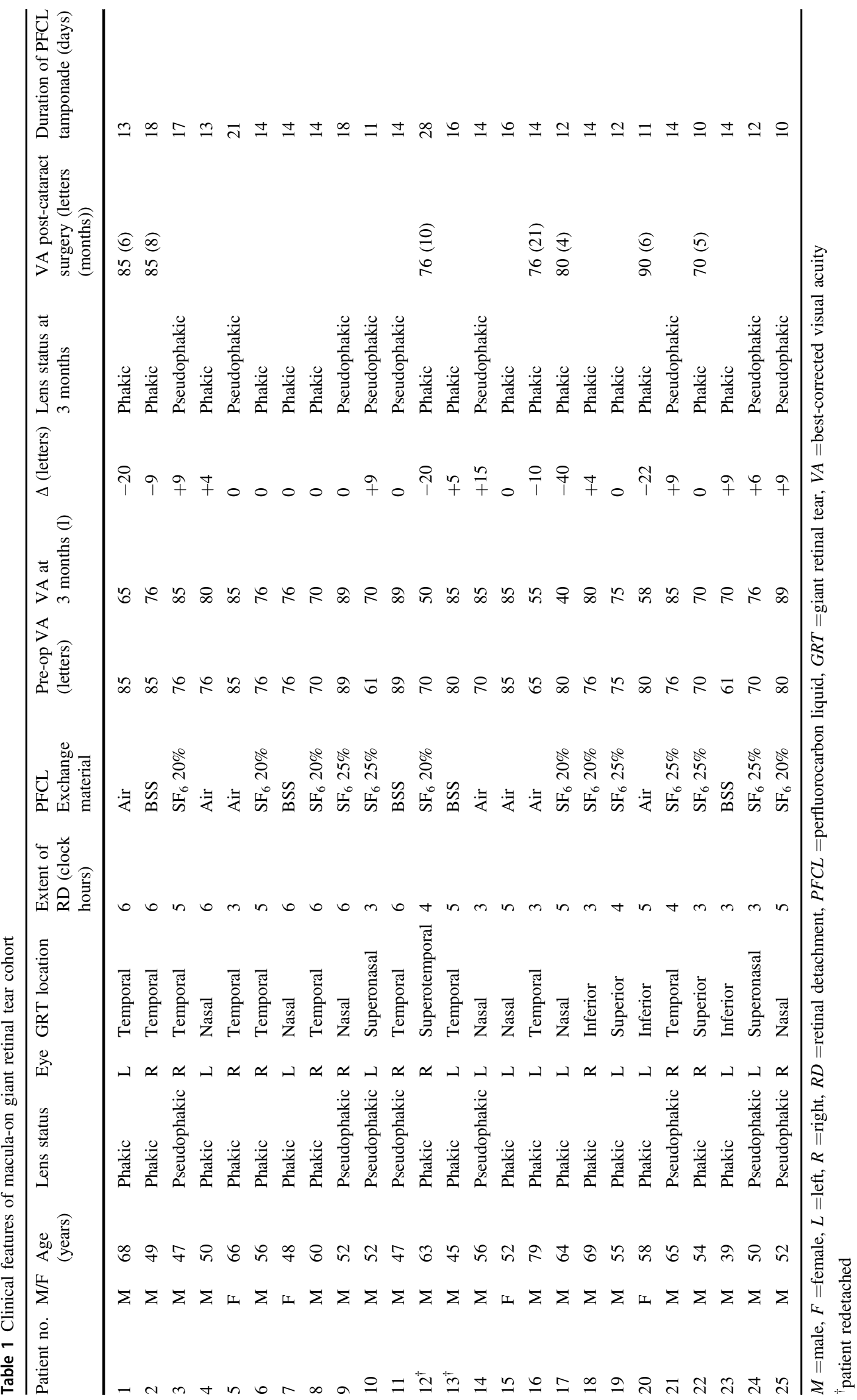


GRT repair was 76 letters, improving to 80 letters after a total of seven patients underwent cataract surgery following a median interval of 6 months (range 4-21 months). This was significantly better than the baseline VA $(p<0.001)$. The prevalence of visual loss $\geq 10$ letters at 3 months was 4 (16\%), which improved to baseline or better in all four following subsequent cataract surgery. Redetachment (maculaon) occurred in two out of 25 patients (8\%) following removal of PFCL due to smaller missed breaks. Both were successfully repaired with cryotherapy and gas tamponade.

In keeping with other reports [5-7], short-term use of post-operative PFCL in this study was associated with good primary and final anatomical success rates. Notably, in contrast to the reported high rate of vision loss after ROSO in the same patient group i.e. macula-on detachments $[1,8$, 9] there were no unexplained visual acuity losses in our series. PFCL may thus mitigate the high incidence of unexplained vision loss after ROSO in those eyes where a good visual outcome is expected. Vitreoretinal surgeons should consider PFCL as an alternative to $\mathrm{SiO}$ or gas as a post-operative tamponade agent in macula-on GRT repair.

Acknowledgements Additional members of the Vitreoretinal Unit of the Royal Victorian Eye and Ear Hospital who performed surgery on these cases: Drs M F McCombe, D Chiu, R Dawkins, E Roufail, J B Clark and D Fabinyi.

Ethical approval The study protocol was approved by the Royal Victorian Eye and Ear Human Research Ethics Committee (HREC approval $07 / 759 \mathrm{H}$ ) and all procedures were conducted in accordance with the tenets of the Declaration of Helsinki 7th revision.

\section{Compliance with ethical standards}

Conflict of interest The authors declare that they have no conflict of interest.

\section{References}

1. Moya R, Chandra A, Banerjee PJ, Tsouris D, Ahmad N, Charteris DG. The incidence of unexplained visual loss following removal of silicone oil. Eye. 2015;29:1477-82.

2. Roca JA, Wu L, Berrocal M, Rodriguez F, Alezzandrini A, Alvira $\mathrm{G}$, et al. Un-explained visual loss following silicone oil removal: results of the Pan American Collaborative Retina Study (PACORES) Group. Int J Retin Vitr. 2017;3:26.

3. Bottoni F, Bailo G, Arpa P, Prussiani A, Monticelli M, de Molfetta V. Management of giant retinal tears using perfluorodecalin as a postoperative short-term vitreoretinal tamponade: a long-term follow-up study. Ophthalmic Surg. 1994;25:365-73.

4. Campbell WG, McCombe MF. The management of giant retinal tears with short-term tamponade with perfluoro-N-octane and transscleral diode photocoagulation. 29th Annual Scientific Meeting of the Royal Australian and New Zealand College of Ophthalmologists, Sydney; 1997

5. Sirimaharaj M, Balachandran C, Chan WC, Hunyor AP, Chang AA, Gregory-Roberts J, et al. Vitrectomy with short term postoperative tamponade using perfluorocarbon liquid for giant retinal tears. Br J Ophthalmol. 2005;89:1176-9.

6. Randolph JC, Diaz RI, Sigler EJ, Calzada JI, Charles S. 25-gauge pars plana vitrectomy with medium-term postoperative perfluoro-noctane for the repair of giant retinal tears. Graefes Arch Clin Exp Ophthalmol. 2016;254:253-7.

7. Eiger-Moscovich M, Gershoni A, Axer-Siegel R, Weinberger D, Ehrlich R. Short-term vitreoretinal tamponade with heavy liquid following surgery for giant retinal tear. Curr Eye Res. 2017;42:1074-8.

8. Tode J, Purtskhvanidze K, Oppermann T, Hillenkamp J, Treumer F, Roider J. Vision loss under silicone oil tamponade. Graefes Arch Clin Exp Ophthalmol. 2016;254(8): $1465-71$.

9. Christensen UC, la Cour M. Visual loss after use of intraocular silicone oil associated with thinning of inner retinal layers. Acta Ophthalmol. 2011;90:733-7.

\title{
Trainee confidence managing ocular trauma
}

\author{
Amy-lee Shirodkar ${ }^{1} \cdot$ Damien CM Yeo $\mathbb{D}^{1} \cdot$ Mr. Gary Shuttleworth ${ }^{2}$
}

Received: 29 November 2018 / Accepted: 7 December 2018 / Published online: 7 January 2019

(c) The Royal College of Ophthalmologists 2019

\author{
$\triangle$ Amy-lee Shirodkar \\ a-1.s@gmx.com \\ Cardiff Eye unit, UHW, Cardiff, UK \\ 2 Ophthalmology, Singleton Hospital, Swansea, UK
}

Morris et al. [1], showed a reduction in serious cases of ocular trauma between 1992 and 2009 by fourfold and there is a real risk that repairing ocular trauma is now so 